\title{
Determining the Distribution of Stochastic Impulses Acting on a High Frequency System through an Analysis of Its Vibrations
}

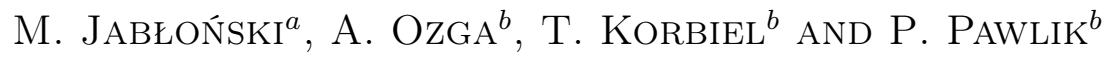 \\ ${ }^{a}$ Faculty of Mathematics and Computer Science, Jagiellonian University \\ Gołębia 24, 31-007 Cracow, Poland \\ ${ }^{b}$ Department of Mechanics and Vibroacoustics, University of Science and Technology \\ al. A. Mickiewicza 30, 30-059 Cracow, Poland
}

The motion of an oscillator with damping excited by impulses has the form $\xi_{\boldsymbol{t}}=\frac{1}{\sqrt{a^{2}-b^{2}}} \sum_{0<t_{i}<t} \eta_{i}$ $\exp \left(-b\left(t-t_{i}\right)\right) \sin \left(\sqrt{a^{2}-b^{2}}\left(t-t_{i}\right)\right)$, where $\xi_{t}$ is the deviation of the oscillator from its balanced position and $t_{i}$ is the time of action of an impulse of the value $\eta_{i}$. Under appropriate assumptions regarding random variables $\left\{t_{i+1}-t_{i}\right\}_{i=1}^{\infty}$ and $\left\{\eta_{i}\right\}_{i=1}^{\infty} \xi_{t}$ is a process which, in the limit as $t$ tends to infinity, is stationary and ergodic. This fact allows us to derive a linear system of equations determining the approximate distribution of variables $\eta_{i}$ whenever the course of the oscillator is known in a sufficiently large interval of time. These equations will be verified in the experiment executed on an electric oscillator RLC of high frequency.

PACS: 45.10.-b, 45.30.+s

\section{Introduction}

The first partial mathematical results regarding vibration of oscillators forced by stochastic impulses and suggesting their possible technological applications can be found in the following papers [1-10].

Let us consider the differential equation of the forced harmonic oscillator with damping

$$
\frac{\mathrm{d}^{2} x}{\mathrm{~d} t^{2}}+2 b \frac{\mathrm{d} x}{\mathrm{~d} t}+a^{2} x=f(t),
$$

where $0<b<a$. The solution of the above equation satisfying the following initial conditions:

$$
x(0)=0 \quad \text { and } \quad \dot{x}(0)=0
$$

has the form

$$
x(t)=\frac{1}{\sqrt{a^{2}-b^{2}}} \int_{0}^{t} \frac{f(u) \sin \left(\sqrt{a^{2}-b^{2}}(t-u)\right)}{\exp (b(t-u))} \mathrm{d} u .
$$

If $\eta_{i}$ is any sequence of real numbers, $t_{i}$ is any increasing sequence of real numbers and $f(t)$ is given by

$$
f(t)=\sum_{t_{i}<t} \eta_{i} \delta_{t_{i}}
$$

where $\delta_{t_{i}}$ are $\delta$-Dirac distributions at $t_{i}$, then the solution of Eqs. (1), (2) takes the following form:

$$
\begin{aligned}
& x(t)=\frac{1}{\sqrt{a^{2}-b^{2}}} \sum_{t_{i}<t} \eta_{i} \exp \left(-b\left(t-t_{i}\right)\right) \\
& \quad \times \sin \left(\sqrt{a^{2}-b^{2}}\left(t-t_{i}\right)\right) .
\end{aligned}
$$

If $\left\{t_{i+1}-t_{i}\right\}_{i=1}^{\infty}$ and $\left\{\eta_{i}\right\}_{i=1}^{\infty}$ form two sequences of ran- dom variables, then $x(t)$ is a process which we denote in the sequel by $\xi_{t}$. If we assume that $\eta_{i}, i=1,2,3, \ldots$, are independent and identically distributed random variables with finite mean value and $\tau_{i}=t_{i}-t_{i-1}, i=1,2, \ldots$, are independent and identically distributed random variables with exponential distribution $F(u)=1-\exp (-\lambda u)$ for $u>0$ and $F(u)=0$ for $u<0$ and for some $\lambda>0$ then, applying to $\xi_{t}$ Theorem 1 from $[11,12]$ with $m=1$, $h_{1}=1$ and function

$$
g=\frac{1}{\sqrt{a^{2}-b^{2}}} \frac{\sin \left(\sqrt{a^{2}-b^{2}} t\right)}{\exp (b t)},
$$

we get the characteristic function of this random variable

$$
\begin{aligned}
\varphi_{t}(s) & =\exp \left(\lambda t \left[\int_{0}^{\infty} \int_{0}^{1}\right.\right. \\
& \left.\left.\exp \left(\frac{\mathrm{i} s y\left(\sin \sqrt{a^{2}-b^{2}} t u\right)}{\sqrt{a^{2}-b^{2}} \exp (b t u)}\right) \mathrm{d} u \varphi_{\eta}(\mathrm{d} y)-1\right]\right) .
\end{aligned}
$$

This function has the limit as $t \rightarrow \infty$, therefore there is a limit of distributions of $\xi_{t}$ as $t \rightarrow \infty$. Similarly we can get that distributions of $\left(\xi_{t_{1}+h}, \xi_{t_{2}+h}, \ldots, \xi_{t_{k}+h}\right)$ are convergent as $h \rightarrow \infty$ for any $k$ and any $t_{1}<t_{2}<\ldots<t_{k}$. This implies that the limiting process $\lim _{s \rightarrow \infty}\left\{\xi_{t}, t>s\right\}$ is stationary, that is, for any $k$, any open subsets $A_{1}, A_{2}$, $\ldots A_{k}$ of $R$ and any $t_{1}<t_{2}<\ldots<t_{k}$ the probability $P\left(\xi_{t_{1}+h}^{-1}\left(A_{1}\right), \xi_{t_{1}+h}^{-1}\left(A_{2}\right), \ldots, \xi_{t_{k}+h}^{-1}\left(A_{k}\right)\right)$ does not depend on $h$. It is also possible to prove that the limiting process $\lim _{s \rightarrow \infty}\left\{\xi_{t}, t>s\right\}$ is ergodic, that is, for almost all $\omega$ in $\Omega \lim _{t \rightarrow \infty} \frac{1}{t} \int_{0}^{t} \xi_{s}(\omega) \mathrm{d} s=\int_{0}^{t} \xi_{0}(\omega) \mathrm{d} \omega$ (the mean value over the space is the same as the mean value over time). 
The characteristic function (7) of $\xi_{t}$ allows us to find stochastic moments of the random variable $\xi_{t}$ of any rate from the following formula:

$$
m_{n}(t)=E\left(x^{n}(t)\right)=\frac{\varphi_{t}^{(n)}(0)}{i^{n}} .
$$

Determining the first derivative of the characteristic function (7) we get

$$
\begin{aligned}
& \varphi_{t}^{\prime}(s)=\varphi_{t}(s) \lambda t \int_{0}^{\infty} \int_{0}^{1}\left(\frac{\mathrm{i} y \sin \sqrt{a^{2}-b^{2}} t u}{\sqrt{a^{2}-b^{2}} \exp (b t u)}\right) \\
& \quad \times \exp \left(\frac{\mathrm{i} s y \sin \sqrt{a^{2}-b^{2}} t u}{\sqrt{a^{2}-b^{2}} \exp (b t u)}\right) \mathrm{d} u \varphi_{\eta}(\mathrm{d} y)
\end{aligned}
$$

and the next derivatives of (7) have the form

$$
\begin{aligned}
& \left(\varphi_{t}(s)\right)^{(n)}=\sum_{j=0}^{n}\left(\begin{array}{c}
n \\
j
\end{array}\right)\left(\varphi_{t}(s)\right)^{(n-j)} \frac{\lambda t \mathrm{i}^{(j+1)} E\left(\eta^{(j+1)}\right)}{\left(\sqrt{a^{2}-b^{2}}\right)^{(j+1)}} \\
& \times \int_{0}^{1}\left(\frac{\sin ^{(j+1)} \sqrt{a^{2}-b^{2}} t u}{\exp ((j+1) b t u)}\right) \mathrm{d} u .
\end{aligned}
$$

Hence, by (8)

$$
\begin{aligned}
& m_{n+1}(t)=\sum_{j=0}^{n}\left(\begin{array}{l}
n \\
j
\end{array}\right) m_{n-j}(t) \frac{\lambda t E\left(\eta^{(j+1)}\right)}{\left(\sqrt{a^{2}-b^{2}}\right)^{(j+1)}} \\
& \times \int_{0}^{1}\left(\frac{\sin ^{(j+1)} \sqrt{a^{2}-b^{2}} t u}{\exp ((j+1) b t u)}\right) \mathrm{d} u .
\end{aligned}
$$

Integrating and taking the limit as $t \rightarrow \infty$ we get

$$
m_{n+1}=\sum_{j=0}^{n}\left(\begin{array}{l}
n \\
j
\end{array}\right) m_{(n-j)} \frac{\lambda E\left(\eta^{(j+1)}\right)}{c^{2+j}} C(j+1),
$$

where for $j>1$ and for $j$ even $C(j)=$ $\frac{j !}{\prod_{r=0}^{j / 2-1}\left((j b / c)^{2}+(2 r)^{2}\right)} \frac{c}{j b}$, for odd $j$

$$
C(j)=\frac{j !}{\prod_{r=0}^{(j-1) / 2-1}\left((j b / c)^{2}+(2 r+1)^{2}\right)}
$$

and $m_{k}$ are the moments of the limiting process $\lim _{s \rightarrow \infty}\left\{\xi_{t}, t>s\right\}$. From (12) for $n=0$ we have $\lambda=\frac{m_{1} c^{2}}{E(\eta) C(1)}$. Substituting it into (12) for $n>0$ we obtain

$$
\begin{aligned}
& m_{n+1}=\sum_{j=0}^{n}\left(\begin{array}{c}
n \\
j
\end{array}\right) m_{(n-j)} \frac{E\left(\eta^{(j+1)}\right)}{c^{2+j}} \\
& \times C(j+1) \frac{m_{1} c^{2}}{C(1) E(\eta)} .
\end{aligned}
$$

Let us assume that $\eta_{i}$ takes only a finite number of values. Multiplying both sides of Eq. (13) by $E(\eta)$ and substituting $E(\eta)=\sum_{i=1}^{k} \eta_{i} p_{i}$ we have

$$
\sum_{i=1}^{k} p_{i}\left[\left(m_{n} m_{1}-m_{n+1}\right) \eta_{i}\right.
$$

$$
\left.+\sum_{j=1}^{n}\left(\begin{array}{l}
n \\
j
\end{array}\right) m_{(n-j)} m_{1} \eta^{(j+1)} \frac{C(j+1)}{C(1) c^{j}}\right]=0 .
$$

Set

$$
\begin{aligned}
& a_{n i}=\left(m_{n} m_{1}-m_{n+1}\right) \eta_{i} \\
& +\sum_{j=1}^{n}\left(\begin{array}{l}
n \\
j
\end{array}\right) m_{(n-j)} m_{1} \eta^{(j+1)} \frac{C(j+1)}{C(1) c^{j}}
\end{aligned}
$$

for $n=1,2, \ldots, k-1$ and $i=1,2, \ldots, k$ and

$$
a_{k i}=1
$$

for $i=1,2, \ldots k$. To find $p_{i}$ it is enough now to solve the following equation:

$$
A P=Y,
$$

where $Y=(0,0, \ldots, 0,1)$ and $P=\left(p_{1}, p_{2}, \ldots, p_{k}\right)$. In practice we can calculate the approximate values $m_{k}$ taking the mean value of the $k$-th powers of the measured oscillator's positions and using the fact that the limiting process $\lim _{s \rightarrow \infty}\left\{\xi_{t}, t>s\right\}$ is ergodic and stationary, that is using the formula

$$
E\left(\xi_{t}^{n}\right) \cong \frac{1}{k} \sum_{i=1}^{k} \xi_{t i / k}^{n}
$$

which is valid for large $t$ and $k$.

In many cases the random variable $\eta$ is a continuous one. If it is so we can find aproximation of the distribution of $\eta$ whenever $\eta$ is bounded. It is enough to take numbers $x_{1}, x_{2}, \ldots, x_{k}$ uniformly displayed in the interval of the values assumed by $\eta$ and find probabilities corresponding to $x_{1}, x_{2}, \ldots, x_{k}$ by (17).

The purpose of this paper is to verify theoretical computation by experiment executed on electric oscillator RLC of high frequency.

\section{Description of the experiment}

In order to test the possible practical applications of the theoretical considerations electromechanical analogies were used and in the experiment it was an RCL system consisting of capacity $C=2 \times 470 \mu \mathrm{F}$ and inductivity $L=3.3 \mathrm{mH}$ that was studied. The measuring unit comprised a measuring computer NI PXI-1031 by National Instruments and a measuring card NI PXI-6251.

The forcing signal $\eta$ is generated on the analogue output of the card at the sampling rate of $1 \mathrm{MHz}$, with simultaneous recording of the system's response on the analogue input. The application was built in Labview 7.1 environment, the source code of the software executing the examination is presented in Fig. 1.

Calibration of the system showed that the current in the circuit, generated by the forcing system satisfies the linear equation with constant coefficients only in certain conditions - for the input function values of $0.4-0.5 \mathrm{~V}$. The RLC system was set in oscillation with impulses that assume values of independent random variables of identical distribution with a finite expected value (Fig. 2). 
The algorithm of the program takes into account that the distribution of probability of the random variable representing the distance between the impulses is exponential. It has also been taken into account that the distances between the impulses and the impulse values are probabilistically independent. The impulses were executed with the help of single samples of the shortest executable duration of $1 \times 10^{-6} \mathrm{~s}$, issuing from the sampling rate.
The parameters of the RCL system were selected so that the circuit allowed for the vibrations for which the value of the current periodically equals 0 , and the characteristic values of the oscillator, namely, its damping coefficient $b=104200$ and the frequency of free vibrations $a=309305$ were computed with the help of Matlab software and cftool function - CurveFitting Toolbox.

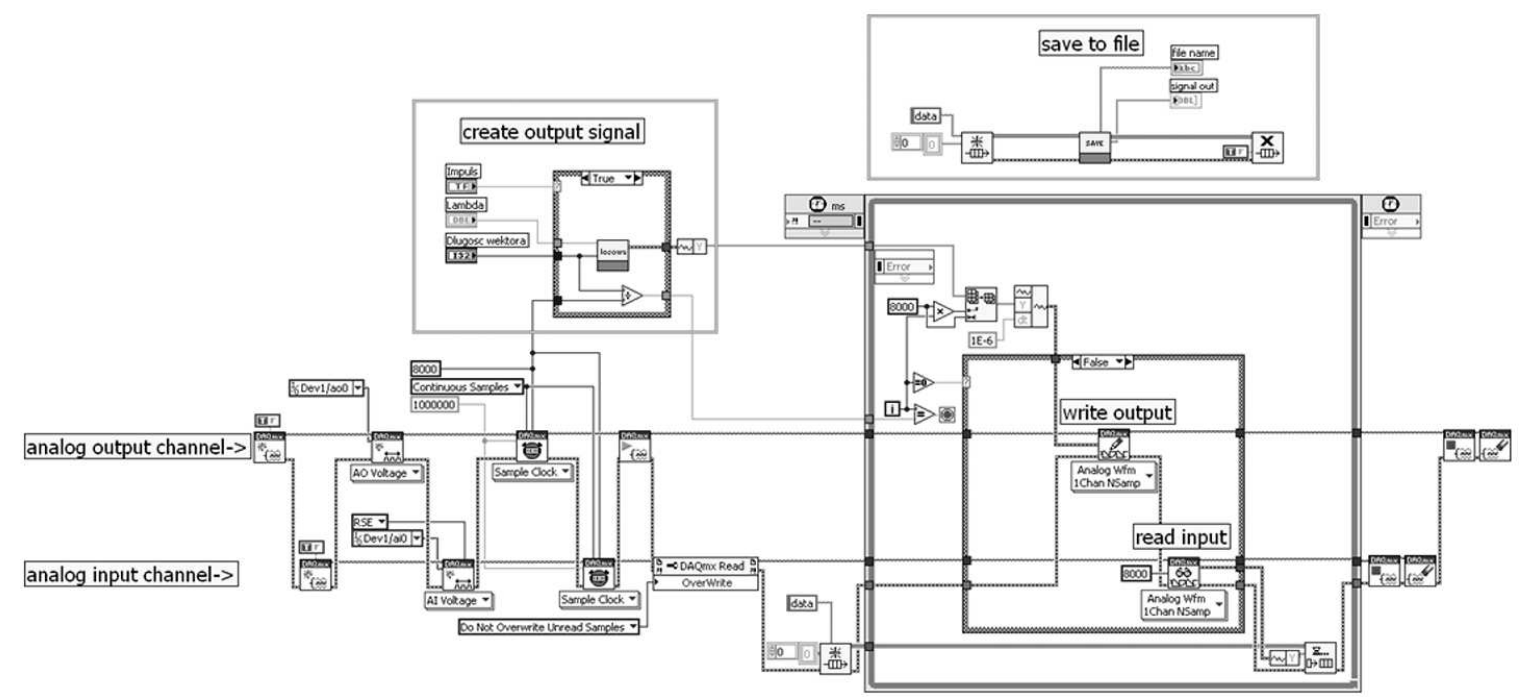

Fig. 1. A diagram of the vibration generator and the measuring algorithm.

\section{Results of the experiment}

In the experiment, the RLC system with the parameters listed above was excited with impulses of the values $\eta \in\{3077,3867\}$. These impulses correspond with the values $0.4 \mathrm{~V}$ and $0.5 \mathrm{~V}$ produced by the generator for the period of $10^{-6} \mathrm{~s}$. Three distributions of the variable $\eta$ were tested. The results have been presented in Table.

In the search for correspondence between a theoretical system and a physical one, the experiments and simulation were carried out on a system whose oscillations were excited with the help of three different cases of distribution $\eta \in\{3077,3867\}$, corresponding with the impulses of the values $0.4 \mathrm{~V}$ and $0.5 \mathrm{~V}$. The experiments and simulations provided the results presented in Table.

As the results of the simulation and experiment differ insignificantly from the assigned values, we can conclude that the experiment has confirmed the validity of theoretical formulae.

\section{Conclusions}

In order to confirm experimentally that the developed mathematical model describes correctly the functioning of an actual physical system, an experiment was carried out on a high frequency RLC system. In spite of

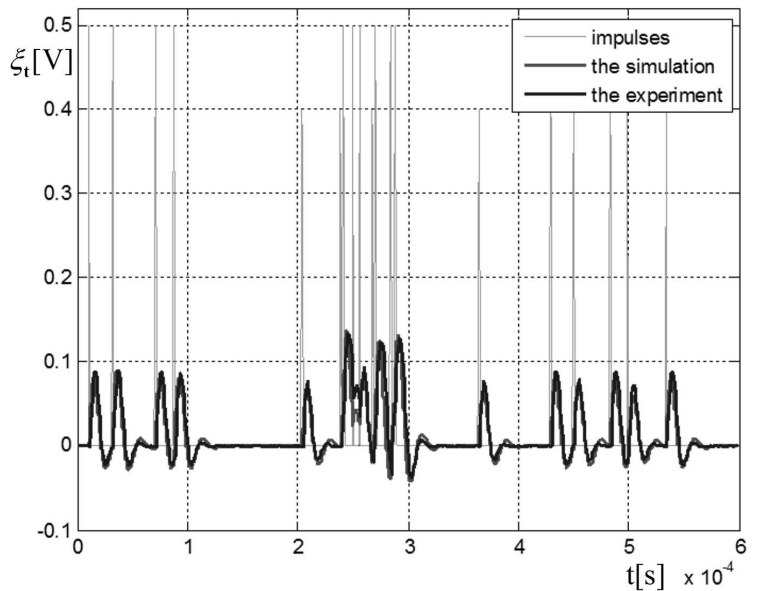

Fig. 2. Comparison of the results of experimental simulation of oscillation evoked by the distribution $P(0.4 \mathrm{~V})=1 / 2, P(0.5 \mathrm{~V})=1 / 2$.

certain instability of this system and the problems connected with limited memory and power of the computer, an experimental proof of correctness of the mathematical model was obtained. Therefore, the possibility of application of the model in practice has been confirmed. It must be remembered, however, that in practice the 
formulae will require certain corrections and adjustment due to the fact that the conditions in which the systems are to be used will not satisfy precisely the mathematical assumptions. At this moment it is possible to extend our considerations to the string, and certain results of this extension can be found in [13].

Determination of distributions of stochastic impulses forcing an RLC system.

TABLE

\begin{tabular}{|c|c|c|c|c|}
\hline & \multicolumn{2}{|c|}{ Experiment } & \multicolumn{2}{|c|}{ Simulation } \\
\hline $\begin{array}{l}\text { Assigned distribu- } \\
\text { tions of stochastic } \\
\text { impulses }\end{array}$ & $\begin{array}{l}\text { Stochastic mo- } \\
\text { ments determined } \\
\text { from the movement } \\
\text { trajectory of the } \\
\text { oscillator }\end{array}$ & $\begin{array}{l}\text { Distributions of } \\
\text { stochastic impulses } \\
\text { forcing the RLC } \\
\text { system determined } \\
\text { from equations }\end{array}$ & $\begin{array}{l}\text { Stochastic mo- } \\
\text { ments determined } \\
\text { from the movement } \\
\text { trajectory of the } \\
\text { oscillator }\end{array}$ & $\begin{array}{l}\text { Distributions of } \\
\text { stochastic impulses } \\
\text { forcing the RLC } \\
\text { system determined } \\
\text { from equations }\end{array}$ \\
\hline $\begin{array}{l}\text { I distribution } \\
P(3077)=1 / 2 \\
P(3867)=1 / 2\end{array}$ & $\begin{array}{l}m_{0}=1 \\
m_{1}=0.0221 \\
m_{2}=0.0022 \\
m_{3}=2.1481 \times 10^{-4} \\
m_{4}=2.5856 \times 10^{-5}\end{array}$ & $\begin{array}{l}P(3077)=0.5196 \\
P(3867)=0.4804\end{array}$ & $\begin{array}{l}m_{0}=1 \\
m_{1}=0.0215 \\
m_{2}=0.00217 \\
m_{3}=2.20223 \times 10^{-4} \\
m_{4}=2.91632 \times 10^{-5}\end{array}$ & $\begin{array}{l}P(3077)=0.5134 \\
P(3867)=0.4866\end{array}$ \\
\hline $\begin{array}{l}\text { II distribution } \\
\begin{array}{l}P(3077)=1 / 3 \\
P(3867)=2 / 3\end{array}\end{array}$ & $\begin{array}{l}m_{0}=1 \\
m_{1}=0.0220 \\
m_{2}=0.0023 \\
m_{3}=2.2807 \times 10^{-4} \\
m_{4}=2.7745 \times 10^{-5}\end{array}$ & $\begin{array}{l}P(3077)=0.3221 \\
P(3867)=0.6779\end{array}$ & $\begin{array}{l}m_{0}=1 \\
m_{1}=0.02235 \\
m_{2}=0.00234 \\
m_{3}=2.4684 \times 10^{-4} \\
m_{4}=3.40013 \times 10^{-5}\end{array}$ & $\begin{array}{l}P(3077)=0.3333 \\
P(3867)=0.6667\end{array}$ \\
\hline $\begin{array}{l}\text { III distribution } \\
\begin{array}{l}P(3077)=1 / 4 \\
P(3867)=3 / 4\end{array}\end{array}$ & $\begin{array}{l}m_{0}=1 \\
m_{1}=0.02245 \\
m_{2}=0.00238 \\
m_{3}=2.37536 \times 10^{-4} \\
m_{4}=2.91407 \times 10^{-5}\end{array}$ & $\begin{array}{l}P(3077)=0.2633 \\
P(3867)=0.7367\end{array}$ & $\begin{array}{l}m_{0}=1 \\
m_{1}=0.02268 \\
m_{2}=0.002418 \\
m_{3}=2.59269 \times 10^{-4} \\
m_{4}=3.64027 \times 10^{-5}\end{array}$ & $\begin{array}{l}P(3077)=0.2421 \\
P(3867)=0.7579\end{array}$ \\
\hline
\end{tabular}

\section{Acknowledgments}

This work has been financed by the Polish Ministry of Science and Higher Education, research project No. N N501 180636.

\section{References}

[1] R. Iwankiewicz, S. Nielsen, J. Sound Vibr. 156, 407 (1992).

[2] R. Iwankiewicz, in: Proc. 10th IFIP, WIG 7.5 Working Conf. on Reliability and Optimization of Structural Systems 2002, Eds. H. Furuta, M. Dogaki, M. Sakano, Balkema, Osaka (Japan) 2003, p. 103.

[3] R. Iwankiewicz, Meccanica 37, 12 (2000).
[4] S.O. Rice, Bell System Techn. J. 23, 1 (1944).

[5] J.B. Roberts, J. Sound Vib. 2, 336 (1965).

[6] J.B. Roberts, J. Sound Vib. 2, 375 (1965).

[7] J.B. Roberts, J. Sound Vib. 24, 23 (1972).

[8] J.B. Roberts, J. Sound Vib. 28, 93 (1973).

[9] J.B. Roberts, P.D. Spanos, Int. J. Non-Linear Mech. 21, 111 (1986).

[10] L. Takác, Acta Math. Hung. 5, 203 (1954).

[11] M. Jabłoński, A. Ozga, Mechanics 25, 156 (2006).

[12] M. Jabłoński, A. Ozga, Acta Phys. Pol. A 118, 74 (2010).

[13] M. Jabłoński, A. Ozga, Arch. Acoust. 34, 601 (2009). 